\title{
Familienbewusste Arbeitsbedingungen - (K)ein Thema für Betriebs- und Personalräte?
}

\author{
Tanja M. Brinkmann \\ Rena Fehre
}

Die Vereinbarkeit von Erwerbs- und Privatleben spielt bei der Wahl einer Arbeitgeberin bzw. eines Arbeitsgebers für fast alle Beschäftigten mit Kindern unter 18 Jahren eine ebenso große Rolle wie das Gehalt. Auch für 64 \% der Beschäftigten ohne Kinder gilt dies. Der Beitrag beleuchtet anhand einer empirischen Studie, ob und wie sich Betriebs- und Personalräte für eine bessere Vereinbarkeit von Erwerbs- und Privatleben einsetzen und damit das zukunftsfähige Thema familienbewusste Personalpolitik gestalten.*

\section{1 \\ Problem- und Frage- stellung}

Das Thema Vereinbarkeit von Erwerbsund Privatleben hat auf der politischen Ebene nach wie vor Konjunktur. Die im europäischen Vergleich stark ausgeprägte Kinderlosigkeit von Männern und Frauen in Deutschland wird als wichtiges Indiz für die schwierige Vereinbarkeit von Erwerbstätigkeit und Familie interpretiert. Durch gesetzliche Maßnahmen, wie die Einführung des Elterngeldes im Jahr 2007 oder der geplante Ausbau der Betreuungseinrichtungen für unter dreijährige Kinder, wird versucht, die Lust auf ein Leben mit eigenen Kindern und damit die Geburtenrate zu erhöhen. Die Erwerbstätigkeit von Frauen ist in den letzten Jahrzehnten kontinuierlich angestiegen und wird voraussichtlich weiter wachsen. Nach der Geburt eines Kindes arbeiten Frauen insbesondere in den alten Bundesländern - jedoch häufig nur in Teilzeit, was ebenfalls als Indiz für die schwierige Balance von Erwerbs- und Privatleben und die beharrlich traditionelle Arbeitsteilung zwischen Männern und Frauen interpretiert werden kann.

Familienbewusster bzw. familienfreundlicher sollen auch die Unternehmen werden, in denen abhängig Beschäftigte erwerbstätig sind. Die Bundesregierung bzw. das Bundesministerium für Familie, Senioren, Frauen und Jugend versucht daher, mit Hilfe zahlreicher in Auftrag gegebener Studien (BMFSJ 2005, 2006a) und Handlungshilfen (BMFSJ 2006b, 2007, 2008), den Unternehmen familienbewusste ${ }^{1}$ Personalpolitik schmackhaft zu machen. Darüber hinaus gibt es zahlreiche Initiativen (z. B. Erfolgsfaktor Familie, Lokale Bündnisse für Familie), die durch Beratung, Information oder Vernetzung Unternehmen unterstützen.

Das empirische Wissen, was auf der betrieblichen Ebene im Themenfeld Vereinbarkeit von Erwerbs- und Privatleben gegenwärtig passiert, ist noch gering (Botsch et al. 2006, S. 485). Noch weniger ist über das Handeln von Betriebs- und Personalräten im Themenbereich Vereinbarkeit bekannt. Hierzu soll der folgende Artikel einen Beitrag leisten. Grundlage ist eine qualitative Studie, in der zehn Unternehmen untersucht wurden, die im Bereich familienbewusster Personalpolitik zertifiziert sind. Es werden zwei Fragestellungen fokussiert:

- Welche Bedeutung hat das Thema Vereinbarkeit von Erwerbs- und Privatleben in der Arbeit von Betriebs- und Personalräten in diesen Unternehmen?

- Wie bringen sich Betriebs- und Personalräte in die Gestaltung familienbewusster Personalpolitik ein?

Im Folgenden werden zunächst die Bereiche und Phasen sowie gesetzlichen Mitbestimmungsmöglichkeiten der Interessenvertretungen ${ }^{2}$ herausgearbeitet und der Forschungsstand zum Themenbereich Vereinbarkeit von Erwerbs- und Privatleben in der Arbeit von Betriebs- und Personalräten dargelegt (Abschnitt 2). Nach der Skizzierung des audit berufundfamilie ${ }^{\circledR}$ (Abschnitt 3) und der Darstellung des Methodendesigns (Abschnitt 4) werden zur Beantwortung der beiden Fragestellungen die empirischen Ergebnisse der Studie vorgestellt und mit den Erkenntnissen anderer Untersuchungen verglichen (Abschnitte 5 und 6). Der Beitrag endet mit einem Fazit und Schlussfolgerungen (Abschnitt 7).

\begin{abstract}
Wir haben diesen Artikel mehrmals mit Professor Dr. Helmut Spitzley diskutiert und konnten von seinen kritisch-konstruktiven Anmerkungen profitieren. Er war unser Projektleiter und ist im April 2009 gestorben. Seine wertschätzende, unterstützende und lebensbejahende Art ist nach wie vor in uns lebendig. Wir widmen inm diesen Artikel.

1 Wir präferieren an dieser Stelle den Begriff familienbewusst statt familienfreundlich. Unsere Erfahrungen wie auch andere Studienergebnisse (Wagner 2006, S. 3f.) zeigen, dass der Begriff Familienfreundlichkeit von Unternehmen und zum Teil auch von Akteursgruppen innerhalb eines Unternehmens unterschiedlich definiert wird. Weiterhin sind nicht alle als familienfreundlich deklarierte Maßnahmen auch tatsächlich familienfreundlich, z. B. Arbeitszeitflexibilisierung (Spitzley 2007). Sie können aber dann als familienbewusst bezeichnet werden, wenn sie mit dem Ziel konzipiert und eingeführt werden, privaten Bedürfnissen von Beschäftigten entgegenzukommen und mögliche Interessenkonflikte zwischen Akteursgruppen im Unternehmen überhaupt erst sichtbar zu machen. $\mathrm{Ob}$ familienbewusste Angebote in der Umsetzung tatsächlich familienfreundlich sind und die Vereinbarkeit von Beruf und Familie verbessern, können letztlich nur die Beschäftigten selbst entscheiden (OECD 2001, S. 147)

2 Als Interessenvertretung werden im Folgenden Betriebs- und Personalräte sowie Frauen- und Gleichstellungsbeauftragte bezeichnet.
\end{abstract}

Tanja M. Brinkmann, Dipl. Sozialpädagogin, Soziolgin M. A., Stipendiatin des Promotionskollegs der Hans-Böckler-Stiftung "Geschlechtsverhältnisse im Spannungsfeld von Arbeit, Organisation und Demokratie" an der Universität Marburg. Arbeitsschwerpunkte: Work-Life-Balance und Geschlechterforschung.

e-mail: tanja.brinkmann@staff.unimarburg.de

Rena Fehre, Soziologin M. A., wissenschaftliche Mitarbeiterin am Institut Arbeit und Wirtschaft der Universität Bremen. Arbeitsschwerpunkte: Lebensphasenorientierte Personalpolitik, insbesondere Vereinbarkeit von Beruf und Pflege, demografischer Wandel.

e-mail: rfehre@iaw.uni-bremen.de 


\begin{tabular}{ll}
\hline $\begin{array}{l}\text { Übersicht 1: Beteiligungs- und Mitbestimmungsmöglichkeiten von } \\
\text { Betriebs- und Personalräten in Unternehmen bei familienbewusster } \\
\text { Personalpolitik }\end{array}$ \\
\hline Bereiche und Phasen & Zentrale Fragen \\
\hline Initiative & $\begin{array}{l}\text { Ergreift die Arbeitgeber- oder Interessenvertretungsseite die Initiative, } \\
\text { damit die Vereinbarkeit von Erwerbs- und Privatleben im Unternehmen } \\
\text { auf die Agenda gesetzt wird? }\end{array}$ \\
\hline $\begin{array}{l}\text { Ziel- und } \\
\text { Maßnahmenbestimmung }\end{array}$ & $\begin{array}{l}\text { Welche Ziele werden mit den Angeboten zur Vereinbarkeit von Erwerbs- } \\
\text { und Privatleben verfolgt? } \\
\text { Welche konkreten Maßnahmen brauchen Beschäftigte? } \\
\text { Führt die Interessenvertretung Bedarfserhebungen bei den Beschäftigten } \\
\text { zur Vereinbarkeit von Erwerbs- und Privatleben durch? }\end{array}$ \\
\hline $\begin{array}{l}\text { Welche Rolle und Funktion hat die Interessenvertretung bei der konkreten } \\
\text { Realisierung familienbewusster Personalpolitik? } \\
\text { Initiiert die Interessenvertretung betriebliche Projektgruppen zur Verein- } \\
\text { barkeit von Erwerbs- und Privatleben, die sich der konkreten Umsetzung } \\
\text { der geplanten Maßnahmen widmen oder arbeitet sie in diesen mit? } \\
\text { Gibt es Betriebs- bzw. Dienstvereinbarungen zum Themenbereich Verein- } \\
\text { barkeit von Erwerbs- und Privatleben? } \\
\text { Verbreitet, bewirbt und kommuniziert die Interessenvertretung die } \\
\text { vorhandenen Maßnahmen? }\end{array}$ \\
$\begin{array}{l}\text { Können alle Beschäftigtengruppen (z. B. Frauen und Männer, alle Quali- } \\
\text { fikationsgruppen) die Angebote nutzen? } \\
\text { Wie viele Beschättigte nutzen tatsächlich die angebotenen Maßnahmen } \\
\text { familienbewusster Personalpolitik? }\end{array}$ \\
\hline Nutzung/Evaluation &
\end{tabular}

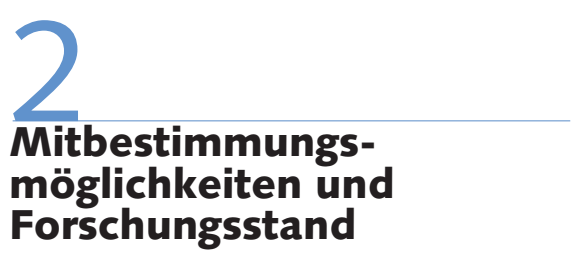

Die Beteiligungs- und Mitbestimmungsmöglichkeiten von Interessenvertretungen bei der Vereinbarkeit von Erwerbs- und Privatleben sind in vier unterschiedlichen Bereichen bzw. Phasen denkbar (Übersicht 1).

Seit der Novellierung des Betriebsverfassungsgesetzes 2001 definiert $\$ 80$ Abs. 1, Ziff. 2b die Vereinbarkeit von Beruf und Familie als Aufgabengebiet von Interessenvertretungen. Betriebsräte können die Initiative ergreifen und familienbewusste Personalpolitik auf ihre Agenda und die des Unternehmens setzen. Die zentrale Frage ist: Ergreifen die Betriebsräte die Initiative oder ist es der Arbeitgeber bzw. die Arbeitgeberin? Auch wenn aus repräsentativen Befragungen bekannt ist, welche Erwartungen Beschäftigte mit betreuungspflichtigen Kindern und Pflegeaufgaben an ein familienfreundliches Unternehmen haben (dazu ausführlich BMFSFJ 2006c; Klenner 2007; Klenner/Schmidt 2007), können die Bedarfe je nach Branche wie auch nach Sozialstruktur des Unternehmens (z. B. Anzahl und Alter der Beschäftigten) stark differieren. Es ist deswegen eine konkrete Zielund Maßnahmenbestimmung (z. B. durch Mitarbeiterbefragung) notwendig, um die Bedarfe der Beschäftigten im Bereich fami- lienbewusster Personalpolitik zu ermitteln. Die konkrete Umsetzung der als notwendig ziellen und arbeitszeitlichen Aufwand (z. B. die Einführung eines Kontakthalteprogramms von Beschäftigten in Elternzeit oder die Etablierung von Telearbeit). Häufig werden in aktiven Unternehmen dazu Projektgruppen eingerichtet. Arbeitet die Interessenvertretung bei einer solchen Projektgruppe mit? Bemüht sie sich um Betriebs- bzw. Dienstvereinbarungen im Bereich familienbewusster Personalpolitik? Familienbewusste Maßnahmen können noch so kreativ und exzellent sein, sie müssen zunächst kommuniziert werden, damit die Beschäftigten über die Möglichkeiten Bescheid wissen und sie nutzen. Auch hier kann die Interessenvertretung aktiv werden und die ausgearbeiteten Angebote aktiv bewerben (z. B. bei Betriebsversammlungen oder in der Betriebsratszeitung). Letztlich bleibt die Frage, ob die angebotenen Maßnahmen von den Beschäftigten genutzt werden und wer sie nutzt (z. B. Hochqualifizierte, Frauen). Aus einer Gerechtigkeitsperspektive ist für die Interessenvertretung relevant, ob alle Beschäftigten Zugriff auf die Angebote haben oder ob sie auf bestimmte Gruppen ausgerichtet sind. Die Nutzung bzw. Evaluation ist notwendig, damit einerseits die Ziel- und Maßnahmenerreichung familienbewusster Personalpolitik überprüft werden kann. Andererseits zeigt der Nutzungsgrad, inwieweit Angebote zur Vereinbarkeit gelebte Praxis im Unternehmen werden. erachteten Maßnahmen erfordert finan-
Vorliegende Forschungsergebnisse verweisen darauf, dass Betriebs- und Personalräte bislang wenig aktiv im Bereich Vereinbarkeit von Erwerbs- und Privatleben sind. In Bezug auf die Initiative zeigen Studienergebnisse widersprüchliche Befunde. Während einige primär die Arbeitgeberseite als Impulsgeber sehen (Döge/Behnke 2006, S. 12f.), beschreiben andere, dass es Interessenvertretungen sind, die das Thema auf die Agenda setzen: „Zwar ist die Mehrheit der Betriebsräte nicht aktiv für Familienfreundlichkeit eingetreten. Aber in den Betrieben, in denen etwas geschieht, sind es am ehesten die Betriebsräte, die Aktivitäten in Gang bringen" (Klenner 2004, S. 282; ebenso Botsch et al. 2006, S. 486). Die derzeit aktuellste Betriebsrätebefragung des Wirtschafts- und Sozialwissenschaftlichen Instituts der Hans-BöcklerStiftung (WSI) aus dem Jahr 2007 zeigt, dass in $59 \%$ aller Betriebe die Betriebsräte das Thema familienfreundliche Arbeitsbedingungen in den letzten zwei Jahren überhaupt nicht thematisiert haben und damit keinerlei Initiative zeigten (Klenner 2008, S. 345). Im Bereich der Ziel- und Maßnahmenplanung bringen sich Interessenvertretungen dagegen - zumindest in Unternehmen mit audit berufundfamilie ${ }^{\circledR}$ - häufig aktiv ein (Döge/Behnke 2006, S. 13f.).

Mit Blick auf die Umsetzung fällt auf, dass Betriebsvereinbarungen zu familienfreundlichen Arbeitsbedingungen eher Seltenheitswert genießen. In der Betriebsrätesondererhebung des WSI zeigt sich, dass im Jahr 2003 gerade einmal 8,4\% aller Betriebe mit mehr als 20 Beschäftigten und Interessenvertretung eine Betriebsvereinbarung zu familienfreundlichen Arbeitsbedingungen hatten (Klenner 2004, S. 279). Im Jahr 2005 war das Niveau ähnlich niedrig (Lindecke 2005, S. 323; Klenner 2007, S. 24). In der aktuellen WSI-Betriebsrätebefragung haben 9,6\% der Betriebe mit mehr als 20 Beschäftigten und Betriebsrat eine Betriebsvereinbarung in diesem Themenbereich (Klenner 2008, S. 343f.). Die Gestaltung familienbewusster Arbeitsbedingungen spielt in der Betriebsratsarbeit, so resümiert Lindecke, „eine eher randständige Rolle“ (Lindecke 2005, S. 326). Klenner vergleicht das Fortschrittstempo im Zeitverlauf in Bezug auf entsprechende Betriebsvereinbarungen mit dem einer "Schnecke“ (Klenner 2008, S. 345).

Ein etwas größeres Engagement zeigen in diesem Themenbereich Personalräte. So verfügen $22,2 \%$ aller öffentlichen Institu- 
tionen über mindestens eine Dienstvereinbarung zu familienfreundlichen Arbeitsbedingungen. Personalräte besuchen öfter Schulungen zu diesem Thema und greifen es häufiger als Betriebsräte in ihrer Arbeit auf (Lindecke 2005, S. 323). Die Wahrscheinlichkeit, dass Betriebs- und Personalräte an dem Themenbereich Vereinbarkeit von Erwerbs- und Privatleben arbeiten, steigt mit der Größe des Betriebes (Klenner 2004, S. 281). Mit anderen Worten: Beschäftigte in kleinen und mittelgroßen Unternehmen haben seltener eine Interessenvertretung, die das Thema Vereinbarkeit von Erwerbs- und Privatleben auf ihre Agenda setzt. Unternehmen mit Interessenvertretung stehen insgesamt jedoch besser da als Unternehmen ohne gewählte Vertretungen. Beblo/Wolf weisen nach, „dass die Wahrscheinlichkeit von Chancengleichheit fördernden und familienfreundlichen Maßnahmen in Betrieben mit Betriebsrat deutlich höher ist" (Beblo/Wolf 2004, S. 566).

Über die Nutzung bzw. Evaluation von Angeboten familienbewusster Personalpolitik ist empirisch wenig bekannt, insbesondere welche Rolle Interessenvertretungen dabei spielen. Wagner verweist darauf, dass vor allem Beschäftigte mit unterer oder mittlerer Qualifikation und primär Frauen die Maßnahmen in Anspruch nehmen, Hochqualifizierte und Führungskräfte aber nur schwer erreichbar sind (Wagner 2006, S. 9f.).

\section{3 \\ Zum Zertifikat audit berufundfamilie ${ }^{\circledR}$}

Die in unserer Studie untersuchten Unternehmen sind aufgrund ihrer familienbewussten Personalpolitik mit dem audit berufundfamilie ${ }^{\circledR}$ zertifiziert. Das audit berufundfamilie ${ }^{\circledR}$ geht auf eine Initiative der Gemeinnützigen Hertie-Stiftung zurück, die dieses Instrument Mitte der 1990er Jahre entwickelt hat. Das Audit erfasst zunächst die im Unternehmen bereits angebotenen Maßnahmen zur besseren Vereinbarkeit von Erwerbs- und Privatleben und ermittelt das betriebsindividuelle Entwicklungspotenzial. In zwei Workshops werden Strategien und Zielvereinbarungen mit konkreten Zielen und Maßnahmen erarbeitet, die innerhalb von drei Jahren verwirklicht werden sollen. Dabei werden acht
Handlungsfelder familienbewusster Personalpolitik bearbeitet: Arbeitszeit, Arbeitsorganisation, Arbeitsort, Informationsund Kommunikationspolitik, Führungskompetenz, Personalentwicklung, Entgeltbestandteile/geldwerte Leistungen und Service für Familien. Mindeststandards in Bezug auf die Qualität oder Quantität der Maßnahmen sind nicht vorgegeben. An dem zweiten sogenannten Auditierungsworkshop nimmt ein repräsentativer Querschnitt der Beschäftigten des Unternehmens teil. Für die von der Geschäftsführung unterzeichnete Zielvereinbarung werden die Unternehmen mit einem Zertifikat ausgezeichnet. Sie müssen im Folgenden jährlich einen Zwischenbericht verfassen, in dem sie verdeutlichen, wie sie mit der Umsetzung der geplanten Maßnahmen vorankommen. Wollen die Unternehmen das Zertifikat behalten, müssen sie sich nach drei Jahren einer Re-Auditierung unterziehen.

Das audit berufundfamilie ${ }^{\circledR}$ ist derzeit das in Deutschland am stärksten verbreitete strategische Managementinstrument familienbewusster Personalpolitik. Unter Schirmherrschaft des Bundesministeriums für Familie, Senioren, Frauen und Jugend und des Bundesministeriums für Wirtschaft und Technologie wird es von arbeitgeber- und arbeitnehmerbezogenen Dachorganisationen empfohlen. Im April 2009 waren knapp 700 Unternehmen und Institutionen in Deutschland mit dem Audit zertifiziert.

\section{4 Methodisches Vorgehen}

Zehn der zertifizierten Unternehmen sind Teil unserer Studie. ${ }^{3}$ Es handelt sich also um einen sehr selektiven Anteil von Unternehmen bzw. Betriebs- und Personalratsgremien, die, quantitativ betrachtet, eher selten in Deutschland zu finden sind. Im Gegensatz zu Unternehmen ohne das audit berufundfamilie ${ }^{\circledR}$ sind diese Unternehmen gezwungen, systematisch und zeitlich stringent familienbewusste Personalpolitik auszubauen, um das Zertifikat nicht zu verlieren.

Die Studienergebnisse basieren auf 20 Experten- bzw. Expertinneninterviews. Die Konzeption der qualitativen Studie ermöglicht zwei unterschiedliche Perspektiven auf die familienbewusste Personalpolitik des jeweiligen Unternehmens: die Geschäftsführungs- bzw. Projektleitungs- und die Interessenvertretungsseite. Deshalb wurden sowohl Interessenvertreter als auch Geschäftsführer bzw. Personalverantwortliche befragt. Beiden Seiten wurden in den Interviews im Wesentlichen die gleichen Fragen gestellt. Ziel dieser Vorgehensweise war es, der betrieblichen Wirklichkeit möglichst nahe zu kommen sowie Unterschiede und Gemeinsamkeiten bei der Wahrnehmung und Bewertung familienbewusster Personalpolitik herauszuarbeiten. ${ }^{4}$

Weiterhin stellten die Unternehmen den Interviewerinnen vor den Befragungen die Zielvereinbarungen und Zwischenberichte im Rahmen des audits berufundfamilie $^{\circledR}$ zur Verfügung. Dabei zeigte sich, dass die Ziele und Maßnahmen unternehmensspezifisch unterschiedlich sind. Sie werden vor allem von den Ideen, Bedarfen, Strukturen und Möglichkeiten im Unternehmen abgeleitet. Gemeinsam ist den untersuchten Unternehmen jedoch, dass sie Maßnahmen in mindestens sieben von insgesamt acht der oben genannten Handlungsfelder verwirklicht haben bzw. verwirklichen wollen.

\section{D) \\ (Un)Bedeutsamkeit des Themas Vereinbarkeit von Erwerbs- und Privatleben}

In den untersuchten Unternehmen waren es nicht die Interessenvertretungen, die das audit berufundfamilie ${ }^{\circledR}$ angeschoben haben. Das heißt, die Initiative, am Audit teilzunehmen, kam fast ausnahmslos von Seiten der Geschäftsführung. Dieser Befund wird durch quantitative Forschungsergebnisse in auditierten Unternehmen bestätigt (Döge/Behnke 2006, S. 12f.; Gerlach et al.

\footnotetext{
3 Dies waren drei Großunternehmen (über $500 \mathrm{Be}$ schäftigte), fünf mittelständische (100-500 Beschäftigte) und zwei kleine Unternehmen (weniger als 100 Beschäftigte). Sechs der zehn Unternehmen sind privatwirtschaftlich, zwei Non-Profit-Unternehmen und zwei öffentliche Institutionen.

4 So stellen beispielsweise Botsch et al. (2006, S. 486) die unterschiedliche Bewertung der Betriebsparteien bei der familienbewussten Personalpolitik heraus. Beile und Jahnz zeigen, dass sich die AuBendarstellung von Work-Life-Balance-Maßnahmen (z. B. im Internet oder in Geschäftsberichten) deutlich von der gelebten betrieblichen Realität im Unternehmen unterscheidet (Beile/Jahnz 2007, S. 99)
} 
2007, S. 17f.). Dass die Geschäftsführungen aktiv das Audit anschieben, könnte daran liegen, dass sie das Zertifikat auch aufgrund von Image- und externen Marketingzwecken (z. B. verbesserte Rekrutierung neuer Beschäftigter) anstreben. Die Interessenvertretungen verhalten sich - so unser Befund - beim Zustandekommen der Auditierung entweder passiv oder wohlwollend unterstützend. Was aber fördert dann die Initiative, familienbewusste Personalpolitik auf die Unternehmensagenda zu setzen? Nicht selten wird davon berichtet, dass eine subjektive Betroffenheit hinsichtlich des Vereinbarkeitsproblems eine förderliche Wirkung gehabt habe. Weiterhin wird durch einen gestiegenen Frauenanteil und durch Hochqualifizierte, die als Fachkräfte gehalten werden sollen, das Thema Vereinbarkeit von Erwerbsund Privatleben auf die Agenda genommen.

Wenn die Initiative nicht von den Interessenvertretungen kommt, stellt sich die Frage, welche Bedeutung das Thema Vereinbarkeit von Erwerbs- und Privatleben in der Arbeit des Interessenvertretungsgremiums überhaupt hat. Döge/Behnke fanden hierzu heraus: „Vereinbarkeitspolitik gehört deutlich nicht zum Kerngeschäft von Personal- und Betriebsräten; die Beachtung und Verankerung des Themas im Betriebs- oder Personalrat ist stark von engagierten Einzelpersonen abhängig. Sie sind es in der Regel, die den Betriebs- oder Personalrat im Audit vertreten und wiederum die gewonnenen Informationen in die Arbeitnehmervertretung hineintragen" (Döge/Behnke 2006, S. 29f.). In unserer Studie kommen wir zu ähnlichen Erkenntnissen.

Zunächst ist aufschlussreich, wer die Personen sind, die von den Interessenvertretungen als Interviewpartner benannt wurden. Bei der Interviewverabredung wurde immer nach der Person des Betriebs- bzw. Personalratsgremiums gefragt, die für das Thema Vereinbarkeit von Erwerbs- und Privatleben zuständig ist bzw. die beim Auditierungsprozess und der Umsetzung der Ziele und Maßnahmen mitgewirkt hat oder mitwirkt. Das hatte zur Folge, dass nur in drei Fällen Betriebs- bzw. Personalratsvorsitzende die Interviewpartner waren, die auch jeweils eine zeitliche Freistellung für ihre Interessenvertretungsarbeit hatten. Die restlichen Interviewten der Interessenvertretungsseite haben - mit einer Ausnahme - gar keine oder nur eine sehr geringe zeitliche Freistellung. Das heißt, ein Großteil der im Themenfeld Vereinbarkeit aktiven Personen verfügt über geringe zeitliche Ressourcen und mutmaßlich auch über begrenzte Durchsetzungsmöglichkeiten innerhalb des eigenen Gremiums.

Auffällig ist auch, dass die Interviewten häufig zwischen sich selbst und den anderen Personen des Betriebs- bzw. Personalrats unterscheiden. Bei der Frage „Welche Rolle spielt das Thema Vereinbarkeit von Beruf und Familie innerhalb Ihres Betriebs- bzw. Personalrätegremiums?" antworten sie zunächst häufig nur für sich selbst, dass ihnen persönlich das Thema sehr wichtig sei.

Im Verlauf der Interviews verdeutlicht sich, dass sie innerhalb ihres Gremiums häufig eine Position als Außenseiter oder Einzelkämpfer innehaben, da nicht alle Interessenvertretungsmitglieder das Thema so wichtig wie sie selbst nehmen. Dass sie selbst das Thema bedeutsam finden, hängt mit einer biografiebedingten Betroffenheit der Interviewten zusammen oder mit einer Neugierde gegenüber dem neuen Thema. Einzelkämpfer bzw. -kämpferin für das Vereinbarkeitsthema innerhalb des Interessenvertretungsgremiums $\mathrm{zu}$ sein, ist nicht selten mit Enttäuschungen verbunden, da die Leistung innerhalb der gesamten Interessenvertretung wenig anerkannt wird.

Welchen Raum nimmt das Thema familienbewusste Personalpolitik in der Arbeit des gesamten Interessenvertretungsgremiums ein? Hier zeigt sich eine große Bandbreite. Zwei interviewte Interessenvertretungen betonen die hohe Bedeutung des Themas in ihrer alltäglichen Interessenvertretungsarbeit. Die Mehrheit der Interviewten hebt jedoch die geringe Bedeutung des Themas hervor.

Eine Ursache, warum familienbewusste Personalpolitik häufig eine geringe Bedeutung beigemessen wird, sind Restrukturierungs- und Reorganisationsprozesse und deren Auswirkungen auf Beschäftigung und Arbeitsbedingungen. Sie haben einschneidende Folgen für die betroffenen Belegschaften und werden von Betriebsund Personalräten prioritär behandelt. Die geringe Bedeutung des Vereinbarkeitsthemas hat aber auch den Grund, dass es bei dem Thema „noch keine Konfliktsituationen gegeben hat" (Interview 5, Abs. 51).

Familienbewusste Personalpolitik und das zeigt sich in fast allen der von uns befragten Unternehmen - ist ein Thema, bei dem es zwischen Geschäftsführungsund Interessenvertretungsseite harmoniert. ${ }^{5}$ Die Interviewten dieser Studie heben hervor, dass andere Themenbereiche innerhalb der Interessenvertretungsarbeit viel spannungs- und konfliktbeladener und damit auch zeit- und arbeitsaufwändiger sind. Selbst die beiden Interessenvertretungen, die ihr Verhältnis zur Geschäftsführung in der Zusammenarbeit generell als eher schwierig beurteilen, betonen die gute Zusammenarbeit beim Themenfeld familienbewusster Personalpolitik.

\section{Gestaltungs- und Mitwirkungsmöglichkeiten der Interessenvertretungen}

Im Folgenden soll die zweite Fragestellung näher beleuchtet werden: Welche Gestaltungsmöglichkeiten haben Interessenvertretungen bei der familienbewussten Personalpolitik? Oder anders formuliert: Wird ihnen von der Geschäftsführungsseite in irgendeiner Form die Beteiligung verwehrt? Der Weg zum Zertifikat im Rahmen des audits berufundfamile ${ }^{\circledR}$ führt über die oben beschriebenen Workshops, bei denen die Strategien und die konkreten Ziele und Maßnahmen familienbewusster Personalpolitik vereinbart werden, die in den folgenden drei Jahren im Unternehmen verwirklicht werden sollen.

Alle Interessenvertretungen betonen, dass sie bei der Bestimmung der Ziele und Maßnahmen familienbewusster Personalpolitik uneingeschränkte Mitwirkungsmöglichkeiten hatten. In diesem Anfangsstadium sind für die Interessenvertretungen also Gestaltungsmöglichkeiten gegeben. Die beteiligten Interessenvertretungen nutzen ihre Chance und sind auch mit den Ergebnissen der Workshops zufrieden, bei denen die Ziele und Maßnahmen für die

\footnotetext{
5 Auch bei den quantitativen Ergebnissen von Döge/ Behnke beurteilen die befragten Interessenvertretungen das Verhältnis zur Geschäftsführung als eher konstruktiv (Döge/Behnke 2006, S. 9). Dagegen heben Botsch et al. aufgrund ihrer qualitativen Betriebsfallstudie hervor: „Familienfreundlichkeit ist ein umkämpftes Terrain und ein von vielschichtigen, teils gegensätzlichen Interessen der Betriebsparteien geleitetes Thema" (Botsch et al. 2006, S. 486).
} 
kommenden drei Jahre erarbeitet werden. $\mathrm{Zu}$ einem ähnlichen Ergebnis kommen auch Döge/Behnke (2006, S. 13f.).

In Bezug auf den Prozess der Umsetzung, in dem die geplanten Ziele und Maßnahmen familienbewusster Personalpolitik realisiert werden müssen, zeigt sich diese Mitwirkung nicht in gleicher Weise. Hier werden drei Gruppen von Interessenvertretungen deutlich. Die erste, sehr kleine Gruppe würde sich gern mehr an der Zielund Maßnahmenumsetzung familienbewusster Personalpolitik beteiligen, Projektverantwortliche bremsen ihr Engagement und ihre Mitwirkung jedoch aus. Der Umsetzungsprozess familienbewusster Personalpolitik erfolgt in diesen Fällen als TopDown-Management, bei dem weder die Beschäftigten noch die Interessenvertretung systematisch eingebunden sind.

Die zweite und anzahlmäßig größte Gruppe könnte sich intensiv am Umsetzungsgeschehen beteiligen, tut es aber nicht. Hier werden von Seiten der Projektverantwortlichen und der Geschäftsführung Mitwirkungsmöglichkeiten eingeräumt, diese werden aber von den Interessenvertretungen kaum genutzt. Diese Interviewten betonen, dass ihnen „keine Steine in den Weg gelegt" werden (Interview 5, Abs. 49). Für die praktisch nicht genutzte Möglichkeit der Mitwirkung und -gestaltung nennen die Interessenvertretungen mehrere Gründe. Das Hauptargument ist Zeitnot. Vereinbarkeit von Erwerbs- und Privatleben ist in der Arbeit von Interessenvertretungen nicht vorrangig. Weiterhin pflegen einige Interessenvertretungen ein Rollenselbstverständnis, dass sie zwar reaktiv auf Anfragen reagieren (z. B. Beschwerde eines einzelnen Mitarbeiters über einen Konflikt mit Vorgesetzten beim Betriebsrat), aber selten proaktiv gestaltend wirken. Anders ausgedrückt: Im Rollenselbstverständnis dieser Interessenvertretungen haben die Betriebs- und Personalräte keine Bringschuld.

Die dritte, sehr kleine Gruppe ist wie in den Phasen der Ziel- und Maßnahmenplanung weiterhin aktiv und beteiligt sich kontinuierlich am Umsetzungsprozess. Die Angehörigen dieser Gruppe arbeiten beispielsweise bei Bedarfserhebungen zur Kinderferienbetreuung oder bei der Vorbereitung und Durchführung eines Familientages des Unternehmens mit.

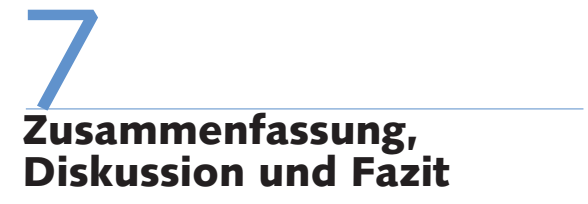

Die Bedeutsamkeit des Themas Vereinbarkeit von Erwerbs- und Privatleben für abhängig Beschäftigte wird durch zahlreiche Studien belegt. Denn unter den nach wie vor defizitären strukturellen Gegebenheiten in Deutschland für Beschäftigte mit betreuungspflichtigen Kindern und/oder Pflegeaufgaben ist die Vereinbarkeit von Erwerbs- und Privatleben häufig ein schwieriger Balanceakt. Eine repräsentative Befragung von abhängig Beschäftigten im Alter von 25-39 Jahren zeigt, wie bedeutsam das Thema ist. 2007 stimmten $92 \%$ der Beschäftigten mit Kindern unter 18 Jahren der Aussage zu „Bei der Wahl eines neuen Arbeitgebers spielt für mich die Frage der Vereinbarkeit von Beruf und Familie eine wichtigere oder ebenso wichtige Rolle wie das Gehalt". Auch Beschäftigte ohne Kinder bejahen diese Aussage zu $64 \% .78$ \% aller Beschäftigten mit Kindern unter 18 Jahren würden sogar für eine bessere Vereinbarkeit von Erwerbs- und Privatleben die Arbeitgeberin bzw. den Arbeitgeber wechseln. ${ }^{6}$ Dem stehen Studienergebnisse gegenüber, die deutlich machen, dass Interessenvertretungen die Bedeutsamkeit, Gestaltbarkeit und Politikfähigkeit dieses strategischen und querschnittsorientierten Themas noch nicht ausreichend erkannt haben.

Die hier vorgestellten Befunde sind auf der Basis einer qualitativen Studie in zehn Unternehmen entstanden, die mit dem audit berufundfamilie ${ }^{\circledR}$ ausgezeichnet sind. Sie sind damit nicht repräsentativ und können lediglich vertiefte Erkenntnisse über das Wirken von Interessenvertretungen in auditierten Unternehmen geben. Die Initiative, sich als Unternehmen am audit berufundfamilie ${ }^{\circledR}$ zu beteiligen und damit die Etablierung und Weiterentwicklung familienbewusster Personalpolitik voranzutreiben, kommt in den zehn Unternehmen fast ausschließlich von der Geschäftsführungsseite.

Die Bedeutung des Themas Vereinbarkeit von Erwerbs- und Privatleben ist bei den interviewten Interessenvertretungen sehr unterschiedlich. Während es bei einigen Interessenvertretungen eine zentrale Rolle spielt, ist es bei der Mehrheit der Interessenvertretungen eher ein „Randthema“, das im Vergleich zu anderen Themen schnell „hinten runter kippt“. Häufig sind die im Rahmen dieser Studie befragten und sich für das Vereinbarkeitsthema engagierenden Interessenvertreterinnen und -vertreter Einzelkämpfer und Außenseiter innerhalb des Gremiums. Sie selbst schätzen die Relevanz des Themas hoch ein, das Gremium platziert und bearbeitet aber andere Themen mit mehr Nachdruck. Dies zeigt sich auch daran, dass die interviewten Interessenvertretungen seltener Betriebsbzw. Personalratsvorsitzende, sondern einzelne Betriebs- bzw. Personalratsmitglieder waren, die für ihre Interessenvertretungsarbeit nur teilweise und gar nicht freigestellt sind. Anders ausgedrückt: Vereinbarkeit von Erwerbs- und Privatleben wird eher selten zur Chefsache erklärt, sondern innerhalb des Betriebs- oder Personalratsgremiums nach unten delegiert. Prinzipiell scheint es, dass auch in auditierten Unternehmen ein Großteil der Betriebs- und Personalräte noch für familienbewusste Personalpolitik gewonnen werden muss, um die Diskrepanz bezüglich der Bedeutung des Themas zwischen Beschäftigten und ihren Interessenvertretungen zu minimieren. Ein unternehmensübergreifendes Betriebs- und Personalrätenetzwerk könnte hier perspektivisch das Engagement der Interessenvertretungen stärken, indem sie noch stärker für das Thema sensibilisiert und qualifiziert werden ${ }^{7}$ und gleichzeitig voneinander lernen können, wie andere Interessenvertretungen das Thema in ihrem Unternehmen voranbringen, auf welche Hindernisse sie stoßen und welche Erfolge sie haben. Solch ein Interessenvertretungsnetzwerk könnte auch dazu beitragen, dass Betriebs- bzw. Personalräte eigenständige und selbstbewusste Gestaltungsoptionen gegenüber der Geschäftsführung vertreten.

Inwieweit bringen sich die Interessenvertretungen während der Maßnahmenund Zielplanung und während des anschließenden Umsetzungsprozesses ein? Und inwieweit ist ihr Engagement von der

\footnotetext{
6 Quelle: http://www.erfolgsfaktor-familie.de/ default.asp?id=14 [Zugriff: 20.4.09]

7 Das DGB-Projekt "Vereinbarkeit von Familie und Beruf gestalten! “ setzt insbesondere auf den Qualifizierungsaspekt von Betriebs- und Personalräten (www.familie.dgb.de). Das Archiv Betriebliche Vereinbarungen der Hans-Böckler-Stiftung zeigt Gestaltungsmöglichkeiten und praktische Beispiele von Betriebsvereinbarungen zur Familienfreundlichkeit (http://www.boeckler.de/73692_81895. html, vgl. auch Maschke/Zurholt 2006).
} 
Geschäftsführung gewollt? Alle Interessenvertretungen waren bei den Workshops zur Erlangung des Zertifikats und damit bei der Maßnahmen- und Zielplanung uneingeschränkt beteiligt. Diese Gestaltungsmöglichkeit nutzen die Interessenvertretungen und sind zufrieden mit den Schwerpunkten und Ergebnissen der geplanten familienbewussten Personalpolitik, die im Rahmen der Zielvereinbarung verabredet wurde. Während ein Teil der Interessenvertretungen noch aktiv an der Umsetzung der Ziele und Maßnahmen familienbewusster Personalpolitik mitwirkt, nutzt ein weitaus größerer Teil der Interessenvertretungen seine Mitgestaltungsmöglichkeit nicht. Die Gründe hierfür sind entweder zeitlicher Art. Oder es drückt sich hier ein Rollenselbstverständnis als Betriebsrat bzw. Betriebsrätin aus, lediglich auf Anfrage auf herangetragene Probleme zu reagieren und nicht selbst familienbewusste Angebote in der Belegschaft zu bewerben. Und nicht selten steht die Setzung anderer thematischer Prioritäten im Vordergrund. Andere Prioritäten, von denen größerer Druck auszugehen scheint, sind insbesondere Entgelt, Reorganisation oder Maßnahmen bei Personalreduzierung.

Nur in wenigen Unternehmen ist das Engagement der Betriebs- bzw. Personalräte im Umsetzungsprozess lediglich be- grenzt erwünscht. In den meisten Unternehmen werden den Interessenvertretungen von Seiten der Geschäftsführung keine Steine in den Weg gelegt, Planung und Realisierung familienbewusster Personalpolitik mitzugestalten. Die Studienergebnisse zeigen vielmehr, dass die Interessenvertretungen in auditierten Unternehmen viel mehr gestalten könnten, wenn sie dem Thema Vereinbarkeit von Erwerbs- und Privatleben ausreichend Raum geben würden. Die Betriebs- und Personalräte unseres Samples werden im Themenbereich familienbewusste Arbeitsbedingungen nicht ausgebremst, sondern bremsen sich selbst aus. Beim Themenkomplex familienbewusste Personalpolitik zeigt sich insgesamt eine kooperative und produktive Zusammenarbeit zwischen Geschäftsführungsund Interessenvertretungsseite, wenn sich Letztere zu einem Engagement entschlossen hat. Selbst die wenigen Interessenvertretungen, die ihr Verhältnis zur Geschäftsführung prinzipiell als eher schwierig einstufen, betonen, dass beim Themenfeld Vereinbarkeit von Erwerbs- und Privatleben eine gute Zusammenarbeit mit der Geschäftsführung vorherrscht. Bei der familienbewussten Personalpolitik in auditierten Unternehmen handelt es sich, so lässt sich schlussfolgern, in der Zusammenarbeit von Interessenvertretung und
Geschäftsführung um ein konsensfähiges und konfliktarmes Thema.

Das aber ändert nichts am Gesamteindruck: Auch in Unternehmen, die sich aufgrund der Zertifizierung explizit mit familienbewusster Personalpolitik beschäftigen, wird dieser Themenbereich in der Regel von den dort agierenden Interessenvertretungsgremien eher nachrangig behandelt. Oder wie es Döge/Behnke pointiert formulieren: Die Interessenvertretungen bleiben "Akteure in zweiter Reihe“ (Döge/Behnke 2006, S. 36). Obwohl sie das Instrument audit berufundfamilie ${ }^{\circledR}$ in der retrospektiven Betrachtung als positiv hervorheben, nutzen sie ihre Mitgestaltungsmöglichkeiten während der Umsetzung und Weiterentwicklung der familienbewussten Personalpolitik nicht erschöpfend. Dabei ist der Rückenwind beim Thema Vereinbarkeit derzeit politisch und medial äußerst günstig. Kluge und weitsichtige Betriebs- und Personalräte könnten das zukunftsorientierte Thema familienbewusste Personalpolitik nutzen, um nicht nur auf die veränderten Realitäten und Bedarfe der Beschäftigten (z. B. Doppelverdienerpaare, Väterengagement bei der Kinderbetreuung, Vereinbarkeit von Erwerbstätigkeit und Pflege) einzugehen, sondern sich selbst positiv zu positionieren und zu profilieren.

\section{LITERATUR}

Beblo, M./Wolf, E. (2004): Chancengleichheit und Vereinbarkeit von Familie und Beruf - Faktoren des betrieblichen Engagements, in: WSI-Mitteilungen 10, S. 561-567

Beile J./Jahnz, S. (2007): Work-Life-Balance in der Unternehmenspraxis, in: Sozialwissenschaften und Berufspraxis 1, S. 85-102

Botsch, E./Lindecke, C./Wagner, A. (2006): Familienfreundlicher Betrieb - Ein Blick in die Praxis, in: WSI-Mitteilungen 9, S. 485-491

Bundesministerium für Familie, Senioren, Frauen und Jugend (BMFSFJ) (2005): Betriebswirtschaftliche Effekte familienfreundlicher Maßnahmen. Kosten-Nutzen-Analyse, Berlin

Bundesministerium für Familie, Senioren, Frauen und Jugend (BMFSFJ) (2006a): Unternehmensmonitor Familienfreundlichkeit 2006. Wie familienfreundlich ist die deutsche Wirtschaft? - Stand, Fortschritte, Bilanz, Berlin
Bundesministerium für Familie, Senioren, Frauen und Jugend (BMFSFJ) (2006b): Familienbewusste Personalpolitik. Informationen für Arbeitnehmervertretungen, Unternehmens- und Personalleitungen, Berlin Bundesministerium für Familie, Senioren, Frauen und Jugend (BMFSFJ) (2006c): Erwartungen an einen familienfreundlichen Betrieb. Erste Auswertung einer repräsentativen Befragung von Arbeitnehmerinnen und Arbeitnehmern mit Kindern oder Pflegeaufgaben, Berlin

Bundesministerium für Familie, Senioren, Frauen und Jugend (BMFSFJ) (2007): Informationen für Personalverantwortliche. Familienfreundliche Maßnahmen im Unternehmen, Berlin

Bundesministerium für Familie, Senioren, Frauen und Jugend (BMFSFJ) (2008): Früher beruflicher Wiedereinstieg von Eltern. Ein Gewinn für Unternehmen und ihre Beschäftigten, Berlin 
Döge, P./Behnke, C. (2006): Betriebs- und Personalräte als Akteure familienbewusster Personalpolitik. Handlungsmuster von Personalvertretungen in Unternehmen und Organisationen mit dem audit berufundfamilie, http://www.beruf-und-familie.de/system/cms/data/dl_data/

670ec4e5f6c1b507dcf1f0658c18bb2c/iaiz_brpr.pdf (Zugriff: 20.4.09)

Gerlach, I./Schneider, H./Juncke, D. (2007): Betriebliche Familienpolitik in auditierten Unternehmen und Institutionen, Arbeitspapier 3, Münster Klenner, C. (2004): Gender - Ein Fremdwort für Betriebsräte?, in: WSIMitteilungen 5, S. 277-286

Klenner, C. (2007): Familienfreundliche Betriebe - Anspruch und Wirklichkeit, in: Aus Politik und Zeitgeschichte 34, S. 17-25

Klenner, C. (2008): Gleichstellung und familienfreundliche Arbeitsbedingungen, in: WSI-Mitteilungen 6, S. 342-345

Klenner, C./Schmidt, T. (2007): Familienfreundlicher Betrieb - Einflussfaktoren aus Beschäftigtensicht, in: WSI-Mitteilungen 9, S. 494-501
Lindecke, C. (2005): Geschlechterpolitik im Betrieb, in: WSI-Mitteilungen 6, S. 322-328

Maschke, M./Zurholt, G. (2006): Chancengleich und familienfreundlich. Analyse und Handlungsempfehlungen, Frankfurt a. M.

OECD (2001): OECD-Employment Outlook 2001. http://www.oecd.org/ dataoecd/11/12/2079435.pdf (Zugriff 20.4.09)

Spitzley, H. (2007): Theorie und Empirie der Arbeitszeitflexibilisierung Leitlinien zur Qualitätsverbesserung der betrieblichen Arbeitszeitgestaltung, in: Dilger, A./Gerlach, I./Schneider, H. (Hrsg.): Betriebliche Familienpolitik. Potenziale und Instrumente aus multidisziplinärer Sicht, Wiesbaden, S. 125-140

Wagner, A. (2006): Familienfreundlichkeit - Zum Stand in deutschen Betrieben, http://www.boeckler.de/pdf/v_2006_03_30_wagner_f3.pdf (Zugriff: 20.4.09) 